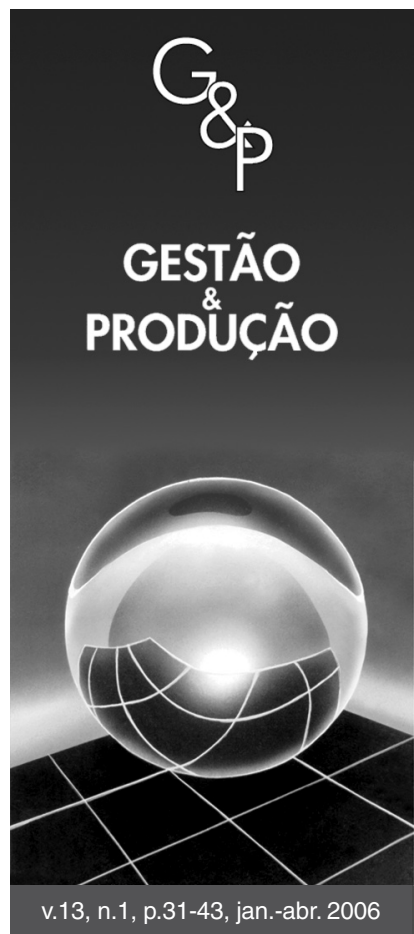

\title{
APRENDIZAGEM CORPORATIVA E ACUMULAÇÃO TECNOLÓGICA: A TRAJETÓRIA DE UMA EMPRESA DE TRANSMISSÃO DE ENERGIA ELÉTRICA NO NORTE DO BRASIL
}

\author{
Anna Carolina Lemos Rosal \\ Paulo Negreiros Figueiredo \\ Escola Brasileira de Administração Pública e de Empresas, EBAPE, \\ Fundação Getulio Vargas, FGV, Praia de Botafogo, 190, \\ CEP 22250-900, Rio de Janeiro, RJ, \\ e-mail: anna.rosal@terra.com.br, pnf@fgv.br
}

Resumo

Este artigo examina, pela perspectiva intra-empresarial, as implicações dos processos subjacentes de aprendizagem para a trajetória de acumulação de capacidades tecnológicas. Esse relacionamento é examinado, à base de um estudo de caso individual, em uma empresa de transmissão de energia elétrica no Norte do Brasil de 1990 a 2004. A métrica para exame da acumulação tecnológica distingue entre capacidades rotineiras (para operar ou usar tecnologias e/ou sistemas de produção existentes) e capacidades inovadoras (para inovar tecnologias elou sistemas de produção). Tais capacidades são examinadas para três funções tecnológicas: i) engenharia, projetos e equipamentos; ii) operação e manutenção; e iii) processos operacionais. A métrica para aprendizagem tecnológica identifica quatro processos examinados à luz de características-chave. O estudo baseia-se em evidências empíricas de primeira mão coletadas a partir de extensivo trabalho de campo. A qualidade da gestão dos vários processos de aprendizagem contribuiu para a maneira e velocidade de construção de tais capacidades na empresa estudada, principalmente a partir do início dos anos 1990. Muito embora haja no Brasil uma ampla retórica, em níveis macro-econômico e macro-político, sobre a importância da inovação tecnológica para o crescimento e desenvolvimento industrial e econômico, há ainda uma escassez de conhecimento sobre o real processo de inovação em nível de empresas. Estudos como este, portanto, podem contribuir para iluminar e apoiar decisões de estratégias empresariais e governamentais para acelerar o desenvolvimento industrial em setores-chave da economia brasileira.

Palavras-chave: acumulação de capacidade tecnológica, processos de aprendizagem, empresas de transmissão de energia elétrica.

\section{Introdução}

Este artigo examina as implicações dos processos de aprendizagem para a maneira e velocidade de acumulação tecnológica, em nível de empresa, na indústria de transmissão de energia. Esse relacionamento é examinado no contexto de economias de industrialização tardia - ou 'em desenvolvimento'. Em meados dos anos 1990, emergiu uma nova geração de estudos sobre aprendizagem tecnológica em empresas de economias em desenvolvimento (ver, por exemplo, Hobday, 1995; Kim, 1998; 1997a; 1997b; Dutrénit, 2000; Figueiredo, 2001; 2003). A partir do início dos anos 1990, emergiram no Brasil alguns estudos empíricos relevantes sobre aprendizagem tecnológica em empresas de diferentes indústrias no Brasil (ver, por exemplo, Tacla e Figueiredo, 2003; Ferigotti e Figueiredo, 2005; Büttenbender, 2005; Ben, 2005; Araujo, 2005).

Adicionalmete, há a necessidade de expandir os estudos empíricos para tipos diversos de empresas no Brasil. 
Isto é importante para gerar entendimento e proporcionar conhecimento sobre o real processo de acumulação tecnológica e de inovação, em nível de empresas, na indústria brasileira. Muito embora haja uma intensa retórica, em nível macroeconômico, sobre a importância da inovação tecnológica para o crescimento industrial brasileiro, muito pouco se avança em termos de medidas que considerem uma perspectiva intra-empresarial para o desenho de estratégias tanto corporativas como, principalmente, governamentais. Por exemplo, empresas da indústria de transmissão de energia elétrica - tão importantes para o crescimento e desenvolvimento industrial regional e nacional - não têm sido estudadas sob essa perspectiva. No entanto, esse tipo de indústria tem recebido atenção de pesquisadores no Brasil, porém sob perspectivas outras que não da aprendizagem tecnológica (ver, por exemplo, Borenstein e Camargo, 1997; Peci, 2000; Palmeira, 2001; Coimbra, 2002; Tolmasquim et al., 2002; Carmo, 2003).

O mais importante ainda é entender esse tema no contexto de economias e/ou regiões de industrialização tardia - ou em desenvolvimento. Empresas que operam nesse contexto caracterizam-se pela necessidade de moverem-se da acumulação de capacidades de mero uso ou operação de sistemas de produção existentes para o acúmulo de capacidades para inovar tecnologias e sistemas de produção (Bell e Pavitt, 1995; Figueiredo, 2001). É nesse contexto que opera a grande maioria de empresas brasileiras. Por isso, o contexto empírico deste estudo é a Eletronorte - Centrais Elétricas do Norte do Brasil -, durante o período de 1990 a 2004.

O termo 'aprendizagem tecnológica' é em geral compreendido em dois sentidos. O primeiro refere-se à trajetória ou o caminho que segue a acumulação de capacidade tecnológica. Essa trajetória pode variar ao longo do tempo, ou seja, a capacidade tecnológica pode ser acumulada em velocidades e direções distintas. O segundo sentido refere-se aos vários processos pelos quais conhecimento técnico é adquirido pelos indivíduos e convertido para o nível organizacional, isto é, conversão de conhecimento tácito individual em capacidades tecnológicas da empresa (Bell, 1984; Figueiredo, 2001; 2003). Neste artigo, aprendizagem é entendida como um processo que permite à empresa acumular (ou não) suas capacidades tecnológicas rotineiras e/ou inovadoras.

A capacidade tecnológica, por sua vez, é definida aqui como os recursos necessários para gerar e gerir aprimoramentos tecnológicos - em processos de produção, produtos e atividades relacionadas a sistemas operacionais e equipamentos. Tais recursos são acumulados e armazenados não apenas em sistemas físicos (máquinas, plantas de produção, software, bancos de dados) e nas mentes de gerentes, engenheiros e técnicos, mas também nas roti- nas, procedimentos, ou seja, no sistema organizacional da empresa (Bell e Pavitt, 1995; Figueiredo, 2001).

Este artigo estrutura-se como segue. Na Seção 2, contém as métricas para acumulação de capacidades tecnológicas e para os processos subjacentes de aprendizagem. O método do estudo subjacente a este artigo é apresentado na Seção 3. Na Seção 4, são apresentados os principais resultados do estudo. Por fim, a Seção 5 discute as conclusões do artigo.

\section{Modelo analítico do artigo}

\subsection{Métrica para exame da acumulação de capacidades tecnológicas}

A trajetória de acumulação de capacidades tecnológicas nas Centrais Elétricas do Norte do Brasil S.A. é examinada à luz da métrica desenvolvida em Figueiredo (2001), adaptada de Lall (1992) e Bell e Pavitt (1995). A métrica adaptada é validada para as características tecnológicas de firmas prestadoras de serviço de transmissão de energia com o auxílio de especialistas da indústria (ver Tabela 1). Para este processo foram envolvidos engenheiros, técnicos e pesquisadores da Eletronorte, do Centro de Pesquisa em Energia Elétrica (CEPEL) e funcionários de outras concessionárias de energia. A métrica faz distinção entre capacidades para usar tecnologias existentes (de rotina) e capacidades inovadoras que criam e aprimoram produtos ou processos e atividades. As capacidades tecnológicas foram divididas em três funções: i) engenharia, projetos e equipamentos: envolvem as capacidades relativas a decisões sobre a evolução tecnológica dos equipamentos e expansão da rede de transmissão, escolha de equipamentos, sistemas e novas tecnologias a serem utilizadas e gestão de projetos; ii) operação e manutenção: envolvem as capacidades para a operação e manutenção do sistema de transmissão, a fim de garantir os serviços com flexibilidade, confiabilidade, continuidade e livre acesso (para a indústria de energia elétrica, essa função é essencial, pois é responsável pelo gerenciamento dos ativos de transmissão, garantindo o funcionamento do serviço de transporte de energia); e iii) processos operacionais: envolvem as ferramentas, técnicas e práticas de gestão que permeiam as diversas funções da empresa.

\subsection{Métrica para exame dos processos subjacentes de aprendizagem}

O exame dos processos de aprendizagem na empresa foi realizado conforme o modelo desenvolvido em Figueiredo $(2001 ; 2003)$ - Tabela 2. Aprendizagem tecnológica é decomposta em dois processos distintos: aquisição e conversão de conhecimento técnico. Os processos de aquisição de conhecimento são ainda divididos em externos e internos, e os processos de conversão baseiam-se na 
Tabela 1. Métrica para mensurar capacidades tecnológicas em empresas transmissoras de energia elétrica.

$\begin{array}{cccc}\begin{array}{c}\text { Níveis de } \\ \text { capacidades }\end{array} & \begin{array}{c}\text { Engenharia, projetos e } \\ \text { equipamentos }\end{array} & \text { Operação e manutenção } & \text { Processos operacionais } \\ \text { tecnologicas } & & & \end{array}$

tecnológicas

\section{Capacidades rotineiras}

\section{Básico}

Disponibilizar equipamentos (ativos) para o transporte de energia. Decisão sobre a localização das SEs e dos trechos das LTs.

Terceirização dos estudos elétricos, Projeto Básico e Projeto Executivo. Contratação de empresa para construção, comissionamento e fiscalização das obras.

Sincronização dos trabalhos de constru- Manutenção pós-quebra. ção civil e instalações de equipamentos.

\begin{tabular}{lll}
\hline 2. Renovado & Conhecimento sobre arranjo de subes- Operação em sistemas isolados. & Sistemas de controle de contrato e \\
& tações. Conhecimento de equipamentos Manutenção preventiva (programa- & rastreamento de documentos. \\
& de alta tensão (de $15 \mathrm{kv}$ a $500 \mathrm{kv}$ ). Dar da). & Uso de ferramentas convencionais \\
& suporte técnico durante a construção e Implantação de centros de manu- para gestão de projetos. \\
& montagem de SEs e LTs. Coordenação tenção nas unidades. & Certificação ISO 9000. \\
& e análise dos projetos elaborados pelas & Definição e utilização de práticas \\
consultoras. & de trabalho. \\
\hline
\end{tabular}

\section{Capacidades inovadoras}

3. Extrabásico

Realização dos estudos elétricos e pro-

Operação em sistemas interligados Identificação e implementação de jeto básico.

Especificação básica dos equipamentos (definição dos requisitos mínimos para os equipamentos). Realização de empreendimentos até 230 kv (nível de tensão). Utilização de equipamentos com pouca sofisticação tecnológica (ex: banco de capacitor shunt e reles eletromecânicos). Sistema de transmissão isolado.

Envolvimento na aquisição de equipamentos (analisar propostas apresentadas pelos fabricantes e analisar os documentos apresentados após a aquisição dos equipamentos).

4. Pré-intermediário Coordenação e Elaboração do Projeto Op Executivo de sistemas acima de 230 kv. são acima de 230 kv.

Estudos sistemáticos para modernização Consolidação das atividades da das LTs e SEs. Promover padronização manutenção com a operação.

dos projetos e especificações técnicas. Manutenção baseada na condição Utilização de equipamentos eletrônicos do equipamento. para proteção, controle, comando e supervisão.

Operação e manutenção em sisteDar suporte técnico durante os ensaios cos de energia. de equipamentos nos fabricantes e na Estudos sistemáticos para redução montagem (ensaios de tipo e especiais). do tempo de recomposição do sisParticipar do comissionamento do em- tema. preendimento. Especificação do sistema de proteção, comando, controle, automação e supervisão. Interligação parcial do sistema elétrico da empresa ao sistema nacional.
Garantia da operação com flexibili- Uso de ferramentas convencionais dade, continuidade e confiabilidade para gestão das unidades operacionais e corporativas.

Definição e monitoramento dos Controle de qualidade de rotina.

custos da operação e manutenção. Comprovação de habilidade técnica para execução de empreendimento de trans-missão de energia (ex: registro técnico no CREA). de acordo com os padrões estabele- melhorias nos processos de suportas). Manutenção por melhoria. Integração dos programas compunhamento dos indicadores tacionais.

Elaboração de política para equi- dores.

pamentos reserva e peças sobressa- Criação de banco de dados para entes (redução do estoque). os projetos, desenhos e especifiOperação do sistema de transmis- cações.

: pessoas, suprimento e financeio.

valiação sistemática de fornecesão até $230 \mathrm{kv}$.

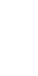


Tabela 1. Métrica para mensurar capacidades tecnológicas em empresas transmissoras de energia elétrica. Continuação.

\begin{tabular}{cccc}
\hline $\begin{array}{c}\text { Níveis de } \\
\text { capacidades } \\
\text { tecnológicas }\end{array}$ & $\begin{array}{c}\text { Engenharia, projetos e } \\
\text { equipamentos }\end{array}$ & Operação e manutenção & Processos operacionais \\
\hline
\end{tabular}

\section{Capacidades inovadoras \\ 5. Intermediário LTs com alta capacidade de transporte (SIL elevado). \\ Planejamento da expansão da rede de transmissão de acordo com o plano nacional (incorporação às incertezas de longo prazo). \\ Executar a coordenação e a aprovação da integração dos sistemas de prote- ção, comando, controle, automação e dos sistemas supervisivos, junto aos fabricantes. \\ Visualizar as interferências entre os empreendimentos. \\ Desenvolvimento de soluções inova-do- ras para problemas específicos da área de concessão da transmissão (ex: PRE - pára-raios energizado, utilização de cabo OPGW em torre Raquet).}

6. Intermediário Implantação de SEs totalmente desassuperior sistidas (utilização de fibra ótica). Elaborar e coordenar a elaboração de especificações com tecnologia digital. Utilização de redes compactas para transmissão com cabo OPGW. Capacidade de desenvolver empreendimentos em locais com variações climáticas, com vegetação e solos diversificados. Interligação total do sistema elétrico da empresa ao sistema elétrico nacional.

7. Avançado Pesquisa e desenvolvimento de novas baseado em tecnologias para redução do custo da pesquisa transmissão.

Digitalização de todo sistema de transmissão.
Estudos sistemáticos para melhoria da operação (busca pela falha zero, redução das perdas de energia e redução das quebras).

Utilização de sistemas eletrônicos de informação para coordenação do funcionamento da rede.
Controle sistemático das práticas de gestão.

Análise crítica do desempenho global utilizado, estabelecendo relação de causa e efeito entre os indicadores. Planejamento participativo com as comunidades envolvidas no empreendimento.

Capacidade de participar e influenciar o planejamento da expansão da rede de transmissão da nação (ex.: indicativo no Brasil: ser membro da CCPE). Certificação ISO 14000.
Utilização de monitores microeletrônicos e técnicas de checagem não destrutivas para avaliação das instalações.

Utilização de aparelhos de controle remoto para inspeção de redes. Substituição da comutação telefônica pela digital para a manutenção. Aproximação da operação nas atividades projetos e engenharia.
Adequação contínua dos processos às alterações do modelo do Setor Elétrico e aos padrões internacionais mais inovadores.
Desenvolvimento de software para P\&D para sistema de gestão. operação e manutenção via P\&D e Desenvolvimento de ferramentas e engenharia.

Operação e manutenção em sistema totalmente digitalizado. metodologias de gestão (software). Desenvolvimento de novos processos via P\&D.

Fonte: Adaptado de Figueiredo (2001).

SE - Subestação de Energia. ONS - Operador Nacional do Sistema. SIL - Search Impedance Level.

LT - Linha de Transmissão. CREA - Conselho Regional de Engenharia e Arquitetura.

CCPE - Comitê de Controle e Planejamento em energia. TPM - Total Produtive Maintenance.

socialização e na codificação de conhecimento. Por meio dessa métrica, podem-se examinar os quatro processos de aprendizagem à luz das seguintes características-chave: variedade, intensidade, funcionamento e interação. As bases conceituais desse modelo, bem como a definição dos quatro processos de aprendizagem e suas características, estão detalhadas em Figueiredo $(2001 ; 2003)^{1}$.

As métricas apresentadas nas Tabelas 1 e 2 são utilizadas, neste artigo, para examinar as implicações dos

1. Os critérios para exame das quatro características-chave dos processos de aprendizagem estão detalhados no estudo subjacente a este artigo (ver Rosal, 2004). processos subjacentes de aprendizagem para a maneira (ou direção) e a velocidade de construção e acumulação de capacidade tecnológica na empresa em estudo. Outros fatores externos e internos ao ambiente da firma podem influenciar a sua trajetória de acumulação de capacidade tecnológica (Kim, 1995; 1997a; 1997b; Figueiredo, 2001). Esses fatores são, entre outros, as políticas públicas, legislação, infra-estrutura, medidas governamentais, condições climáticas e ambientais, condições de mercado e interações com universidades e institutos de pesquisa. Porém, a influências desses fatores sobre a acumulação tecnológica intra-empresarial está além do escopo deste estudo. 
Tabela 2. Métrica para examinar processos de aprendizagem intra-empresariais.

\begin{tabular}{ccccc}
\hline & Variedade & Intensidade & Funcionamento & Interação \\
\hline Processos de & Inexistente/Existente & Uma vez/ & Ruim/Razoável/Bom/ & Fraca/Média/Forte \\
Aprendizagem & (Limitada/ & Intermitente/ & Excelente & \\
& Moderada/Ampla) & Contínuo & & \\
\hline
\end{tabular}

Aquisição externa Existência/inexistência de O uso desse processo pode O modo como um processo é O modo como um processo de conhecimento processos de aquisição de ser contínuo (treinamento criado (critérios para enviar influencia outros procesconhecimento em âmbito anual no exterior para en- indivíduos para treinamento sos internos ou externos de local e/ou externo (por genheiros e operadores), no exterior) e o modo como aquisição de conhecimento exemplo: importação de intermitente ou uma vez ele funciona ao longo do (treinamento no exterior, know-how diferenciado (treinamento esporádico tempo podem fortalecer ou aprendendo na prática) e/ou para a empresa). no exterior). mitigar a variedade e a in- outros processos de convertensidade. Timing: 'aprender são do saber. antes de fazer'.

Aquisição interna Existência/inexistência de O modo como a empresa O modo como um processo é A aquisição interna de code conhecimento processos de aquisição de usa os diferentes proces- criado (centros de pesquisa) nhecimento pode ser deconhecimento mediante sos internos de aquisição e o modo como ele funcio- sencadeada pelo processo atividades internas, que de conhecimento pode in- na ao longo do tempo têm externo de aquisição de copodem ser operacionais, fluenciar a compreensão conseqüências práticas para nhecimento (melhoramenrotineiras e/ou inovadoras que os indivíduos têm dos a variedade e a intensidade. tos na fábrica decorrentes (desenvolvimento de pro- principais envolvidos na Timing: "aprender antes de do treinamento no extedutos). tecnologia. fazer". rior). Isso pode desencadear processos de conversão do saber.

Socialização de Existência/inexistência de A continuidade de pro- O modo como são criados os Incorporação dos diferenconhecimento diferentes processos pelos cessos como o OJT. A mecanismos de socialização tes conhecimentos tácitos quais os indivíduos com- socialização contínua do do conhecimento (treinamen- num sistema viável (estapartilham seu conheci- conhecimento pode con- to interno) e o modo como belecimento de vínculos de mento tácito (reuniões, duzir à codificação do eles funcionam ao longo do saber). A socialização pode solução de problemas em saber. tempo têm consequiências ser influenciada pelos proconjunto $O J T$ ). práticas para a variedade e a cessos externos ou internos intensidade do processo de de aquisição de saber. conversão do saber.

Codificação de conhecimento
Existência/inexistência de A repetição de processos $\mathrm{O}$ modo como é criada a co- O modo como a codificadiferentes processos e me- tais como a padronização dificação do conhecimento e ção do conhecimento é incanismos de codificação das operações. A inexis- o modo como ela funciona ao fluenciada por processos de do conhecimento tácito tência ou intermitência de longo do tempo influenciam aquisição de conhecimento (documentação sistemá- codificação pode restrin- todo o processo de conversão (treinamento no exterior) ou tica, seminários internos, gir a aprendizagem orgaetc.). nizacional. do saber, bem como sua va- por outros processos de soriedade e intensidade. cialização do conhecimento (formação de equipes).

OJT: Treinamento de trabalho (on-the-job training) Fonte: Figueiredo (2001, 2003).

\section{Desenho e método do estudo}

O estudo subjacente a este artigo foi estruturado para examinar as seguintes questões: i) Como se desenvolveu a trajetória de acumulação de competências tecnológicas das Centrais Elétricas do Norte do Brasil S.A., ao longo do período de 1990 a 2004; e ii) Qual foi o papel dos processos subjacentes de aprendizagem intra-organizacional na trajetória de acumulação de capacidades da empresa?

Para descrever a trajetória de acumulação de capacidade tecnológica, foram coletados dados qualitativos e quantitativos relacionados a diversas atividades técnicoorganizacionais e de aprendizagem tecnológica da empresa, durante o período de 1990 a 2004. Isto envolveu a realização de extensivo trabalho de campo no âmbito dessa empresa direcionado à busca de informações sobre capacidade tecnológica e processos de aprendizagem desenvolvidos na empresa. Mais especificamente, foram coletados dados sobre as atividades de engenharia, obras, elaboração e gestão de projetos, processos gerenciais e operacionais, práticas desenvolvidas pela empresa voltadas para as atividades de operação e manutenção do sistema de transmissão, práticas para padronização de projetos e especificação de equipamentos.

Os processos de aprendizagem foram avaliados por meio de dados e informações referentes aos processos e mecanismos de aprendizagem utilizados ao longo do período estudado. As evidências empíricas foram coletadas 
de fontes múltiplas e complementares, como entrevistas formais, reuniões, encontros casuais, consulta a documentos da empresa e observação direta. A Tabela 3 resume as fontes de informação utilizadas para a pesquisa.

Devido às dificuldades inerentes ao questionamento direto sobre a trajetória de acumulação de competências tecnológicas, as perguntas aos entrevistados foram relacionadas às atividades relevantes executadas pela empresa durante o período de 1990 a 2004, às dificuldades encontradas nos projetos e obras, às modificações em equipamentos e às ferramentas/práticas gerenciais. Previamente, foram realizadas conversas iniciais e consultas aos documentos da empresa (contratos de fornecimento), quando foram identificadas as pessoas-chave a serem entrevistadas (funcionários, ex-funcionários e fabricantes de equipamentos). Desta maneira foram selecionadas 19 pessoas que representavam a elite técnica da organização (ver Tabela 4). Alguns funcionários passaram por uma segunda entrevista para confirmação de dados e solução de discrepâncias. Todas as informações coletadas foram organizadas em forma de texto e encaminhadas para validação dos entrevistados.

\section{Principais resultados do estudo}

\subsection{Acumulação de capacidade tecnológica na empresa em estudo}

A Tabela 5 apresenta os tipos e níveis de capacidades encontradas na empresa estudada, bem como o número de anos que foram necessários para acumular níveis específicos dessas capacidades. As células com fundo escuro representam níveis de atividades inovadoras, enquanto as demais representam níveis de capacidade de rotina.

As evidências na Tabela 5 indicam que a empresa acumulou capacidade tecnológica para a função processos operacionais com taxas e velocidades mais lentas do que para as outras funções, levando cinco anos para acumular capacidades inovadoras, sete anos para atingir o nível 4 (pré-intermediário) e mantendo-se no mesmo nível até 2004. O foco inicial da empresa era o de engenharia e equipamentos para transporte de energia. Somente a partir da década de 1990, com a introdução do plano de qualidade total - PQT, a gestão passou a ter mais importância no ambiente interno da empresa. A empresa acumulou capacidades para a função operação e manutenção - alcançando nível 6 (intermediário superior) - mais rapidamente do que para as outras três funções. Na função engenharia, projetos e equipamentos, não chegou a completar o nível 6 de capacidades tecnológicas. Esta função estabilizou-se no nível 5 (intermediário) em função de não se ter realizado a interligação total do sistema de transmissão da empresa ao Sistema Integrado Nacional (atividade relativa ao nível 6).

A acumulação de capacidades para as funções engenharia, projetos e equipamentos e operação e manutenção, em níveis similares, reflete a forte interligação entre essas funções, que são interdependentes. A função processos operacionais não possui relação tão forte com as outras duas funções, contudo, isso não exclui a interação desta função com as demais. Evidências demonstram que o aprofundamento e a manutenção das capacidades tecnológicas das funções engenharia, projetos e equipamentos e operação e manutenção são mais facilmente se-

Tabela 3. Fonte de dados para coleta de evidências na empresa.

\section{Fontes de evidências e técnicas de coleta utilizadas}

1) Entrevistas formais: Entrevistas não estruturadas e semi-estruturadas.

2) Encontros casuais (dentro e fora da empresa).

3) Documentação: Revista da empresa (Corrente-Contínua - várias edições); contratos de fornecimento; editais de licitação; projetos e especificações; manuais e normas internas; dissertações de funcionários da Eletronorte; arquivo técnico; anais de congressos e seminários; e informações de dados de desempenho da manutenção e operação.

4) Observação direta dos indivíduos no trabalho do dia-a-dia da empresa, em treinamento ministrado por fabricantes e durante a realização de ensaios (teste) em equipamentos.

Tabela 4. Relação dos participantes das entrevistas.

\begin{tabular}{lc}
\hline \multicolumn{1}{c}{ Cargo ou função } & Número de entrevistados \\
\hline Assessores da Diretoria & 2 \\
Assistente da Presidência & 1 \\
Engenheiros e técnicos & 5 \\
Superintendentes & 3 \\
Gerentes & 4 \\
Ex-funcionários e consultores & 3 \\
Fabricante de equipamento & 1 \\
\hline
\end{tabular}


Tabela 5. Velocidade de acumulação de competências tecnológicas na empresa em estudo (1990-2004).

\begin{tabular}{|c|c|c|c|}
\hline $\begin{array}{c}\text { Nível de capacidades } \\
\text { tecnológicas }\end{array}$ & $\begin{array}{c}\text { Engenharia, projetos e } \\
\text { equipamentos }\end{array}$ & $\begin{array}{l}\text { Operação e } \\
\text { manutenção }\end{array}$ & Processos operacionais \\
\hline 1) Básico & $\begin{array}{l}\text { Nível atingido antes do } \\
\text { período desta pesquisa. }\end{array}$ & $\begin{array}{l}\text { Nível atingido antes do } \\
\text { período desta pesquisa. }\end{array}$ & $\begin{array}{l}\text { Nível atingido antes do } \\
\text { período desta pesquisa. }\end{array}$ \\
\hline 2) Renovado & $\begin{array}{l}\text { Nível atingido antes do } \\
\text { período desta pesquisa. }\end{array}$ & $\mathrm{n}=0$ & $\mathrm{n}=4$ \\
\hline 3) Extra-básico & $\begin{array}{l}\text { Nível atingido antes do } \\
\text { período desta pesquisa. }\end{array}$ & $\mathrm{n}=4$ & $\mathbf{n}=\mathbf{5}$ \\
\hline 4) Pré-Intermediário & $\mathbf{n}=\mathbf{0}$ & $\mathrm{n}=7$ & $\mathbf{n}=7$ \\
\hline 5) Intermediário & $\mathbf{n}=\mathbf{9}$ & $\mathbf{n}=\mathbf{9}$ & $\begin{array}{l}\text { Nível não atingido de } \\
\text { forma completa. }\end{array}$ \\
\hline 6) Intermediário superior & $\begin{array}{l}\text { Nível não atingido de } \\
\text { forma completa. }\end{array}$ & $\mathbf{n}=\mathbf{9}$ & Nível não atingido. \\
\hline 7) Avançado baseado em pesquisa & Nível não atingido. & Nível não atingido. & Nível não atingido. \\
\hline
\end{tabular}

Fonte: Derivado do estudo empírico.

Nota: $\mathrm{n}=$ número de anos.

dimentadas a partir da execução de atividades inovadoras em processos operacionais.

O exame da trajetória de acumulação de capacidade tecnológica da Eletronorte permite sugerir que a sustentação de níveis inovadores de capacidade tecnológica em determinada função é influenciada pelo modo e velocidade com que são acumuladas as capacidades em outras funções, ou seja, as capacidades tecnológicas tornam-se interdependentes como apontado por Figueiredo (2001) e Tacla e Figueiredo (2003). As capacidades tecnológicas foram acumuladas na Eletronorte não apenas em sistemas físicos e conhecimentos técnicos armazenados nas mentes de seus funcionários, mas especialmente nas várias rotinas e procedimentos da empresa, ou seja, em seu sistema organizacional, o que gerou implicações positivas para o desenvolvimento de atividades inovadoras na empresa (por exemplo, Bell e Pavitt, 1995; LeonardBarton, 1998; Dutrénit, 2000; Figueiredo 2001; Tacla e Figueiredo, 2003).

\subsection{Processos de aprendizagem nas centrais elétricas do norte do Brasil}

\subsubsection{Variedade dos processos de aprendizagem}

De 1990 a 1994 os esforços para a aprendizagem organizacional eram minimamente coordenados. Os principais mecanismos de aprendizagem foram: a contratação de consultores externos para a realização de projetos e a aprendizagem no trabalho 'aprender-fazendo'. Durante esse período, os engenheiros da Eletronorte eram responsáveis pela coordenação e análise dos projetos, atividades que contribuíram para que esses indivíduos desenvolvessem habilidades administrativas e de gestão. A partir de 1993, a empresa deixou de trabalhar com consultores ex- ternos e os recursos humanos internos assumiram a atividade de elaboração dos projetos básicos e executivos, passando a interagir mais fortemente com os fabricantes de equipamentos. No período entre 1995 e 1999, a realização de novos e complexos empreendimentos gerou um aumento no volume de atividades da engenharia. $\mathrm{O}$ contato com os fabricantes era praticamente diário, o que apontou estar bastante associado ao processo de codificação (criação de padrões próprios) e socialização do conhecimento. A aquisição externa de conhecimento por meio do contato com fabricantes foi decisiva para o desenvolvimento de capacidades inovadoras.

As evidências demonstraram que a implantação da ferramenta TPM, em 1996, está associada à criação de vários mecanismos de aquisição externa e interna de conhecimento e a sua codificação e socialização, como, por exemplo, a implantação da matriz de habilidade e conhecimento, que determina a necessidade de treinamento de cada funcionário ou a melhoria nos processos operacionais. A partir de 2000, a diminuição dos investimentos em novos empreendimentos implicou a redução de mecanismos de aprendizagem como a resolução compartilhada de problemas em grupos de trabalho internos e contato com fabricantes. A Tabela 6 resume os principais mecanismos utilizados nos diferentes períodos considerados. A Tabela 7 mostra a variedade dos processos de aprendizagem utilizados pela Eletronorte durante o período de 1990 a 2004.

Apesar de não ter havido aumento representativo nos mecanismos de aquisição externa e interna de conhecimento, os mecanismos de codificação mais do que duplicaram a partir de meados de 1990. Esse fato pode ser relacionado aos esforços de criação de padrões próprios de projetos e à implantação de ferramentas e sistemas gerenciais, que re- 
Tabela 6. Principais processos de aprendizagem utilizados pela empresa estudada.

\begin{tabular}{|c|c|c|c|}
\hline \multirow[t]{2}{*}{ Aquisição externa de conhecimento } & \multicolumn{3}{|c|}{ Períodos } \\
\hline & 1990 a 1994 & 1995 a 1999 & 2000 a 2004 \\
\hline Contratação com consultores externos para realização de projetos & presente & ausente & presente \\
\hline Contrato com consultores externos para implantação de ferramentas de gestão. & presente & presente & ausente \\
\hline Contato com fabricantes nacionais & presente & presente & presente \\
\hline Contato com fabricantes estrangeiros & presente & presente & presente \\
\hline Convênio com universidades & presente & presente & presente \\
\hline Participação em seminários e congressos & presente & presente & presente \\
\hline Participação em grupos de pesquisa & ausente & presente & presente \\
\hline Treinamento externo em Software e língua estrangeira & presente & presente & presente \\
\hline Curso de pós-graduação & presente & presente & presente \\
\hline Aquisição interna de conhecimento & 1990-1994 & 1995-1999 & 2000-2004 \\
\hline Treinamentos internos & presente & presente & presente \\
\hline Por meio de atividades de rotina da empresa "aprender fazendo" & presente & presente & presente \\
\hline Busca & presente & presente & presente \\
\hline Atividades de P\&D & ausente & presente & presente \\
\hline Codificação de conhecimento & 1990-1994 & 1995-1999 & 2000-2004 \\
\hline Codificações e especificações de materiais e sistema & presente & presente & presente \\
\hline Banco de dados para arquivo da engenharia & ausente & presente & presente \\
\hline Criação de padrões de projetos & ausente & presente & presente \\
\hline Apostilas para cursos internos (PQT e TPM) & presente & presente & presente \\
\hline Sistemas de controle gerenciais & ausente & presente & presente \\
\hline Socialização de conhecimento & 1990-1994 & 1995-1999 & $2000-2004$ \\
\hline Soluções compartilhadas de problemas & presente & presente & presente \\
\hline Solução compartilhada de problemas junto com fabricantes & presente & ausente & presente \\
\hline Desenvolvimento de especificações em conjunto com fabricantes & ausente & presente & presente \\
\hline Desenvolvimento de especificações em conjunto com outras áreas da empresa & presente & presente & presente \\
\hline Rotação no trabalho, trabalhos em grupo & presente & presente & presente \\
\hline
\end{tabular}

Fonte: Derivado do estudo empírico.

Tabela 7. Características-chave dos processos de aprendizagem - variedade.

\begin{tabular}{|c|c|c|c|}
\hline \multirow[t]{2}{*}{ Processos de aprendizagem } & \multicolumn{3}{|c|}{ Períodos } \\
\hline & 1990 a 1994 & 1995 a 1999 & 2000 a 2004 \\
\hline Aquisição externa de conhecimento & $\begin{array}{l}6 \\
\text { moderada }\end{array}$ & $\begin{array}{l}7 \\
\text { moderada }\end{array}$ & $\begin{array}{l}7 \\
\text { moderada }\end{array}$ \\
\hline Aquisição interna de conhecimento & $\begin{array}{l}3 \\
\text { limitada }\end{array}$ & $\begin{array}{l}4 \\
\text { moderada }\end{array}$ & $\begin{array}{l}4 \\
\text { moderada }\end{array}$ \\
\hline Codificação de conhecimento & $\begin{array}{l}3 \\
\text { limitada }\end{array}$ & $\begin{array}{l}5 \\
\text { moderada }\end{array}$ & $\begin{array}{l}5 \\
\text { moderada }\end{array}$ \\
\hline Socialização de conhecimento & $\begin{array}{l}2 \\
\text { limitada }\end{array}$ & $\begin{array}{l}5 \\
\text { moderada }\end{array}$ & $\begin{array}{l}5 \\
\text { moderada }\end{array}$ \\
\hline Total de mecanismos & 14 & 21 & 21 \\
\hline
\end{tabular}

Fonte: Derivado do estudo empírico.

sultaram na conversão do conhecimento tácito para o nível organizacional. Esses trabalhos requereram maior interação entre diversas áreas da empresa, entre os engenheiros mais antigos e os recém-contratados e, também, com os fabricantes de equipamentos e bens de capital para energia elétrica, que apresentavam novas soluções. A partir de evidências empíricas, pode-se apontar que, provavelmente, o aumento dos processos de codificação e suas interações com os demais mecanismos de aprendizagem influenciaram positivamente a trajetória de acumulação de capacidades tecnológicas. Isto, por sua vez, alinha-se a estudos anteriores (por exemplo, Tacla e Figueiredo, 2003). 


\subsubsection{Intensidade dos processos de aprendizagem}

A simples incidência de mecanismos de aprendizagem não é suficiente para a construção e acumulação de capacitações tecnológica. A intensidade dos esforços em processos de aprendizagem faz com que algumas práticas sejam incorporadas à rotina da firma, assegurando fluxo constante de conhecimento externo para a empresa e a sua conversão para organização (Bessant, 1998; Figueiredo, 2001). A intensidade nos esforços de aprendizagem foi maior durante o período de 1995 a 1999, quando houve maior aceleração na taxa de acumulação de capacidade tecnológica da empresa nas três funções estudadas. Os processos de aprendizagem foram intensificados, principalmente, devido ao aumento do volume e da complexidade dos empreendimentos realizados pela empresa. Durante esse período, o contato e o compartilhamento de informações com fornecedores eram executados de forma intermitente, praticamente todas as especificações eram feitas com interação de diversos fabricantes, o que parece ter contribuído para que a empresa encontrasse novas soluções para o transporte de energia na Região Norte do País e ter se aprofundado em capacidade tecnológica para engenharia, projetos e equipamentos e para operação e manutenção. A Tabela 8 apresenta a intensidade dos processos de aprendizagem utilizados no período de 1990 a 2004.

Durante esse período, alguns engenheiros da empresa visitaram instalações de transporte de energia em outros países, para conhecer a utilização de novas tecnologias, por exemplo, o PRE - pára-raios energizado. Esta tecnologia foi utilizada como solução para fornecer energia para pequenas comunidades situadas ao longo das linhas de transmissão no Brasil. Foi conhecida a partir de uma viagem à África. Por meio dessa e de outras soluções inovadoras realizadas em conjunto com os fabricantes, a empresa aprofundou ainda mais suas capacidades tecnológicas em engenharia, projetos e equipamentos. A partir de 1996, a intensidade de esforços para os processos de aprendizagem foi multiplicada, em grande parte devido à implantação da metodologia TPM. Praticamente, todos os operadores e técnicos de manutenção foram treinados internamente para a realização de novas tarefas, o que parece ser diretamente relacionado ao desenvolvimento de capacidades inovadoras em operação e manutenção. A partir de 2003 a empresa procurou apenas sustentar seus esforços existentes de aprendizagem. Isto pode estar associado a certas indefinições no campo da legislação regulatória no Brasil em processo de votação parlamentar.

\subsubsection{Funcionamento dos processos de aprendizagem}

De 1990 a 1994 os processos de aprendizagem variaram de ruim a moderado, com algumas exceções em mecanismos isolados, que foram classificados como bom. Os esforços para codificação do conhecimento foram, em muitos casos, inexistentes, sendo que os existentes variaram de ruim para moderado. A ausência de esforços para a codificação de conhecimento teve implicações para o funcionamento de todo processo de conversão de conhecimento durante esse período. As evidências empíricas colhidas por meio das entrevistas com funcionários e exfuncionários da empresa atestam que, até 1994, de modo geral, não havia coordenação e organização de esforços para a aprendizagem e a capacitação dos indivíduos, o que implicou reduzida conversão de conhecimento do nível individual para o nível organizacional. A Tabela 9 mostra como funcionaram os processos de aprendizagem utilizados na empresa ao longo do período estudado.

Tabela 8. Características-chave dos processos de aprendizagem - intensidade.

\begin{tabular}{llll}
\hline Processos de aprendizagem & \multicolumn{1}{c}{ Períodos } \\
& $\mathbf{1 9 9 0}$ a 1994 & $\mathbf{1 9 9 5}$ a 1999 & $\mathbf{2 0 0 0}$ a 2004 \\
\hline Aquisição externa de conhecimento & Descontinuada - baixa & Descontinuada - contínua & Descontinuada \\
Aquisição interna de conhecimento & Descontinuada & Descontinuada - contínua & Descontinuada \\
Codificação de conhecimento & Descontinuada & Contínua & Descontinuada \\
Socialização de conhecimento & Descontinuada & Continuada - descontinuada & Descontinuada \\
\hline
\end{tabular}

Fonte: Derivado do estudo empírico

Tabela 9. Características-chave dos processos de aprendizagem - funcionamento.

\begin{tabular}{llll} 
Processos de aprendizagem & \multicolumn{3}{c}{ Períodos } \\
& $\mathbf{1 9 9 0}$ a 1994 & $\mathbf{1 9 9 5}$ a 1999 & Mo00 a 2004 \\
\hline Aquisição externa de conhecimento & Ruim - Moderado & Moderado - Bom & Moderado \\
Aquisição interna de conhecimento & Ruim - Moderado & Bom - Moderado & Bom - Moderado \\
Codificação de conhecimento & Ruim - Moderado & Bom & Bom - Moderado \\
Socialização de conhecimento & Moderado & Bom
\end{tabular}


No período entre 1995 a 1999, houve melhoria no funcionamento dos processos de aprendizagem. Durante esse período, os mecanismos de aquisição externa e interna de conhecimento variaram entre moderado e bom. O mecanismo de aquisição externa que mais favoreceu o aprofundamento da capacidade tecnológica da empresa foi o contato com os fabricantes. $O$ funcionamento desse mecanismo foi classificado pelos funcionários como bom, pois os fabricantes de equipamentos contribuíram positivamente para processos de elaboração de especificações inovadoras de equipamentos. A partir dos anos 2000, foram evidenciadas praticamente as mesmas classificações para o funcionamento dos processos de aprendizagem, com exceção para solução compartilhada de problemas com fabricantes, que apresentou funcionamento inferior ao período passado, o que pode ter comprometido o aprofundamento das capacidades tecnológicas da empresa, particularmente, para desenvolvimento de habilidades de pesquisa e desenvolvimento.

\subsubsection{Interação dos processos de aprendizagem}

As interações entre os mecanismos de aprendizagem foram estabelecidas a partir dos dados empíricos coletados para este estudo de caso e foram classificadas como fraca, moderada e forte. A Tabela 10 mostra a interação dos processos de aprendizagem utilizados pela Eletronorte durante os períodos de 1990 a 2004.

A estratégia de relacionamento com fabricantes teve forte interação para todo o período desta pesquisa, podendo-se certificar que esse mecanismo foi de extrema relevância para a construção de capacidades tecnológicas da empresa, contribuindo nitidamente para a solidificação dos outros processos de aprendizagem.

\section{Discussões, conclusões e implicações para gestão}

Este artigo enfocou as implicações dos processos subjacentes de aprendizagem para a acumulação de capacidades tecnológicas. Esse relacionamento foi examinado nas Centrais Elétricas no Norte do Brasil no período de 1990 a 2004. Durante este período, foi observada uma diversidade de processos de aprendizagem usados pela empresa que contribuíram para a construção de suas capacidades tecnológicas. $\mathrm{O}$ estudo mostrou que o modo como a empresa gerenciou seus processos de aprendizagem jogou importante papel na maneira e na velocidade que a Eletronorte criou e acumulou capacidades para as diferentes funções tecnológicas. Mais especificamente o estudo encontrou que:

- Em 1990, a empresa já dispunha de capacidades inovadoras em engenharia, projetos e equipamentos. O trabalho de elaboração de projetos por meio de recursos humanos especializados foi o principal mecanismo de aquisição externa de conhecimento para o período até 1993. À Eletronorte cabia, nessa época, apenas coordenar e analisar os projetos. O mecanismo de aquisição interna de conhecimento 'aprender fazendo' foi especialmente relevante para o aperfeiçoamento dessas atividades. Entretanto, esse mecanismo de aprendizagem oferece limite para a acumulação tecnológica a longo prazo (Bell, 1984; Figueiredo, 2001). Os cursos de língua estrangeira também contribuíram para que os engenheiros adquirissem conhecimentos em equipamentos e novas tecnologias, pois, em geral, os manuais eram escritos em língua inglesa e o conhecimento desse idioma abriu um novo canal de comunicação para o contato com os técnicos de outros países;

- O lento desenvolvimento das capacidades tecnológicas da Eletronorte, entre os anos de 1990 a 1994, pode estar associado à falta de investimento e pouca coordenação dos processos de aprendizagem, o que é evidenciado, principalmente, pelas seguintes características: i) limitada aquisição interna e codificação de conhecimento; ii) intensidade baixa/intermitente e funcionamento ruim/ moderado para os processos de aprendizagem; e iii) interação fraca/moderada para os processos de socialização do conhecimento. A limitação dos mecanismos de codificação de conhecimento e o seu funcionamento ruim/moderado prejudicaram a velocidade de transferência de conhecimento entre as diversas unidades da empresa (socialização do conhecimento). Grande parte do conhecimento adquirido por meio dos mecanismos de aquisição externa de conhecimento não era convertido do nível individual para o nível organizacional;

Tabela 10. Características-chave dos processos de aprendizagem - interação.

\begin{tabular}{llll}
\hline Processos de Aprendizagem & \multicolumn{3}{c}{ Períodos } \\
& 1990 a 1994 & 1995 a 1999 & 2000 a 2004 \\
\hline Aquisição externa de conhecimento & Moderada - Fraca & Forte & Forte \\
Aquisição interna de conhecimento & Moderada - Forte & Forte & Forte \\
Codificação de conhecimento & Moderada - Fraca & Forte - Fraca & Forte - Fraca \\
Socialização de conhecimento & Forte & Forte & Forte \\
\hline
\end{tabular}


- Durante o período de 1995 a 1999 a empresa desenvolveu e aprofundou capacidades tecnológicas para as três funções estudadas. Foi aumentada a variedade dos mecanismos de aprendizagem, mas, principalmente, o funcionamento e a intensidade destes foram melhorados e a intensificação dos processos de codificação de conhecimento facilitou a disseminação da aprendizagem no nível organizacional. O maior número dos mecanismos de aprendizagem agregado ao seu melhor funcionamento e intensidade foi especialmente importante para o aprofundamento de capacidades inovadoras em engenharia, projetos e equipamentos e em operação e manutenção. Alinhado aos trabalhos de Dutrénit (2000), Figueiredo (2001) e Tacla e Figueiredo (2003), as evidências sugerem que de 1995 a 1999 a firma foi capaz de acumular diferentes tipos de conhecimento técnicos, o que contribui para explicar a criação e a acumulação de capacidades tecnológicas inovadoras em níveis avançados para as funções engenharia, projetos e equipamentos e operação e manutenção, e a construção de capacidades inovadoras para a função processos operacionais;

- A partir de 2000, com a diminuição dos investimentos no setor elétrico, alguns importantes mecanismos de aprendizagem ficaram comprometidos, como, por exemplo, a interação entre os funcionários da Eletronorte e os fabricantes de equipamentos. Além disso, a empresa não gerou maior variedade nos processos de aprendizagem. Essas evidências podem explicar a estagnação do processo de construção e acumulação de capacidades tecnológicas da empresa ao longo do período de 2000 a 2004. Isto também se alinha às con- clusões de Dutrénit (2000), Figueiredo (2001); Tacla e Figueiredo (2003) sobre os efeitos da estagnação dos esforços de aprendizagem para a acumulação tecnológica na empresa; e

- Portanto, embora aplicando as métricas de capacidade tecnológica e aprendizagem a um tipo de empresa ainda não examinada pela literatura relacionada, este estudo também identificou a gestão dos diversos processos internos de aprendizagem como variável chave para a maneira e velocidade como a empresa acumula suas capacidades tecnológicas.

Obviamente este estudo não contribui para uma generalização estatística, sobre as duas variáveis aqui examinadas, no âmbito da indústria de transmissão de energia elétrica no Brasil. Porém, o intuito é o de aprofundar o entendimento do relacionamento entre essas duas questões em nível intra-empresarial, ou seja, uma generalização analítica. Tal entendimento é importante para se construir conhecimento e contribuir para a aquisição de conhecimento mais preciso sobre o real processo de desenvolvimento tecnológico em empresas de setores industriais estratégicos para o Brasil.

Muito embora haja no Brasil uma ampla retórica, em níveis macroeconômico e macropolítico, sobre a importância da inovação tecnológica para o crescimento e desenvolvimento industrial e econômico no Brasil, notase ainda um fraco conhecimento sobre o real processo de inovação em nível de empresas. Estudos como este, portanto, podem contribuir para clarificar decisões de estratégias empresariais e governamentais voltadas para a aceleração do desenvolvimento industrial em setores estratégicos da economia brasileira.

\section{Referências Bibliográficas}

ARAUJO, R. C. Capacidades tecnológicas e suas implicações para performance técnica: A experiência de duas empresas de telefonia celular no Brasil. 2005. Dissertação de Mestrado - EBAPE, Fundação Getulio Vargas, Rio de Janeiro. 2005.

BELL, M. Learning and the accumulation of industrial technological capacity in developing countries. In: FRANSMAN, M \& KING K. Technological capability in the Third World, London: Macmillan, 1984.

BELL, M \& PAVITT, K. The development of tecnological capabilities. In: Trade, technology and international competitiveness. 1. ed. Waschington, DC, World Bank, 1995.

BEN, F. Acumulação de capacidades tecnológicas e performance técnico-econômica na indústria moveleira: Diferenças inter-empresariais na indústria moveleira no Rio Grande do Sul, Cadernos EBAPE.BR, Edição Especial, Fundação Getulio Vargas, Rio de Janeiro, 2005.
BESSANT, J. Developing continuous improvement capability. International Journal of Innovation Management, v. 2, n. 4, p. 409-429, 1998.

BORENSTEIN, C.; CAMARGO, C. O setor elétrico no Brasil: Dos desafios do passado às alternativas do futuro. Porto Alegre: Sagra Luzzatto, 1997.

BÜTTENBENDER, P. L. Acumulação de competências tecnológicas e os processos subjacentes de aprendizagem na indústria metal-mecânica: A experiência da AGCO - Indústria de Colheitadeiras. Cadernos EBAPE. BR, Edição Especial. Fundação Getulio Vargas. Rio de Janeiro, 2005.

CARMO, B. Planejamento estratégico na Eletronorte no Período de 1996 a 2002 - Implementação e Resultados. Dissertação de Mestrado (Programa de Pós-Graduação em Engenharia de Produção) - Universidade Federal de Santa Catarina, Florianópolis, 2003. 
COIMBRA, J. A utilização do planejamento estratégico na Eletronorte: uma visão processual. 2002. Dissertação de Mestrado (Programa de Pós-Graduação em Engenharia de Produção) - Universidade Federal de Santa Catarina, Florianópolis, 2002.

DUTRÉNIT, G. Learning and knowledge management in the firm: from knowledge accumulation to strategic capabilities. Cheltenham, UK, Northampton, USA. Edward Elgar, 2000.

FERIGOTTI, C. M. S.; FIGUEIREDO, P. N. Managing learning in the refrigerator industry: evidence from a firmlevel study in Brazil. Innovation: Management, Policy and Practice, v. 7, p. 222-239, 2005.

FIGUEIREDO, P. N. Technological learning and competitive performance. Cheltenham, UK, Northampton, USA. Edward Elgar, 2001.

. Learning, capability accumulation and firms differences: evidence from latecomer steel. Industrial and Corporate Change, v. 12, n. 3, p. 607-643, 2003.

HOBDAY, M. Innovation in East Asia: The Challenge to Japan. Aldershot: Edward Elgar, 1995.

KIM, L. The dynamics of Samsung's technological learning in semiconductors. California Management Review, v. 3, n. 39, p. $86-100,1997$ a.

. Imitation to innovation: The dynamics of Korea's technological learning, Boston, MA, Harvard Business School Press, 1997b.

. Crisis construction and organizational learning: Capability building in catching-up at Hyundai Motor. Organization Science, v. 9, p. 506-21, 1998.
LALL, S. Technological Capabilities and Industrialization. World Development, v. 2, n. 20, p. 165-186, 1992.

LEONARD-BARTON, D. Nascentes do Saber: Criando e sustentando as fontes de inovação, Rio de Janeiro, Fundação Getulio Vargas, 1998

PALMEIRA, J. N. Manutenção Produtiva Total: O caso Eletronorte. 2001. (Dissertação de Mestrado). Fundação Getulio Vargas, EBAPE, Rio de Janeiro, 2001.

PECI, A. Impacto de reestruturação e privatização na gestão integrada do setor de energia elétrica: análise do setor a partir de uma abordagem de redes. 2000 (Dissertação de Mestrado) - Fundação Getulio Vargas, EBAPE, Rio de Janeiro, 2000.

ROSAL, A. C. Acumulação de competências tecnológicas e os processos subjacentes de aprendizagem na indústria de transmissão de energia elétrica: $O$ caso das Centrais Elétricas do Norte do Brasil S.A. ELETRONORTE. (Dissertação de Mestrado), Fundação Getulio Vargas, EBAPE, Rio de Janeiro, 2004.

TACLA, C. L.; FIGUEIREDO P. N. Processos de aprendizagem e acumulação de competências tecnológicas: evidências de uma empresa de bens de capital no Brasil. Revista de Administração Contemporânea, v. 3, n. 7, p. 101-126, 2003.

TOLMASQUIM, M; OLIVEIRA, R.; CAMPOS, A. As empresas do setor elétrico brasileiro: Estratégias e performance. Rio de Janeiro: CENERGIA - COPPE/UFRJ, 2002. 


\title{
CORPORATE LEARNING AND TECHNOLOGICAL ACCUMULATION: THE TRAJECTORY OF AN ELECTRICAL ENERGY TRANSMISSION COMPANY IN NORTHERN BRAZIL
}

\begin{abstract}
Based on an intra-company standpoint, this article examines the implications of underlying learning processes in the accumulation of technological capability in an electrical energy transmission company in Northern Brazil from 1990 to 2004. The framework for examining the accumulation of technological capability distinguishes between routine (the use or operation of existing technologies and production systems) and innovative capabilities (the innovation of technologies and/or production systems). These capabilities are examined for three technological functions: i) engineering, design and equipment; ii) operation and maintenance; and iii) operational processes. The framework for technological learning identifies four processes examined in the light of four key characteristics: variety, intensity, functioning and interaction. The study is based on first-hand empirical evidence compiled from extensive field work. The quality of the management of the various learning processes within the company of this case study has contributed to the accumulation of different types of technological capabilities, especially from the early 1990s on. Although the discourse in Brazil on the macro-economic and macro-political levels espouses the importance of technological innovation for industrial growth and development, knowledge about the real process of innovation at a business level is still inadequate. Studies such as this one may therefore contribute to aid and support business and government strategies aimed at accelerating development in key industrial sectors of Brazil's economy.
\end{abstract}

Keywords: accumulation of technological capability, learning processes, electrical energy transmission companies. 
